\title{
Interfaces
}

\section{Le format, principe d'une esthétique contemporaine}

David Zerbib

\section{(2) OpenEdition}

1 Journals

Édition électronique

URL : https://journals.openedition.org/interfaces/3023

DOI : 10.4000/interfaces.3023

ISSN : 2647-6754

Éditeur :

Université de Bourgogne, Université de Paris, College of the Holy Cross

\section{Édition imprimée}

Date de publication : 12 juillet 2021

ISSN : 1164-6225

\section{Référence électronique}

David Zerbib, «Le format, principe d'une esthétique contemporaine », Interfaces [En ligne], 45 | 2021, mis en ligne le 12 juillet 2021, consulté le 15 septembre 2021. URL : http://journals.openedition.org/ interfaces/3023; DOI : https://doi.org/10.4000/interfaces.3023

Ce document a été généré automatiquement le 15 septembre 2021.

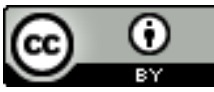

Les contenus de la revue Interfaces sont mis à disposition selon les termes de la Licence Creative Commons Attribution 4.0 International. 


\title{
Le format, principe d'une esthétique contemporaine
}

\author{
David Zerbib
}

\begin{abstract}
« Je m'intéresse à cette notion de format sans savoir véritablement pourquoi. Une des raisons réside peut-être dans le fait que penser en terme de format, c'est penser en terme d'hétérogénéité. Le format permet de produire des allers-retours, des va-et-vient entre différents formats (et je ne parle pas d'introduire ce qu'on appelle de la sousculture) qui ont chacun leurs codes précis. » Boris Achour (Achour, Francblin et Piron, n.p.)
\end{abstract}

\section{Hétérogénéité, transitivité, normativité}

1 Pourquoi s'intéresser à la notion de format? Il y a dans les intuitions de l'artiste contemporain Boris Achour, qui s'exprimait dans un entretien au sujet du jeu des formats dans son œuvre, plusieurs idées importantes qui permettent d'introduire cette question sur un plan théorique. La première fait signe vers une pensée de l'hétérogénéité ; la seconde concerne une logique de transitivité, de passage, de traversée ou de traduction déhiérarchisée (les «allers-retours » qui mettent sur le même plan différentes formes culturelles); la troisième implique une question de normativité relative et située (chaque format possédant ses propres codes). Hétérogénéité, transitivité, normativité : ces trois dimensions de l'objet de notre questionnement suffiraient à justifier un intérêt théorique. Ces éléments invitent en effet à une réflexion sur la question de l'œuvre et sa manière d'opérer à partir de certaines conditions techniques. La notion de format, interrogée ainsi depuis un champ de l'art contemporain où viennent résonner des questions qui le débordent largement, met la pratique artistique en relation avec d'importants enjeux culturels, sociaux, politiques, scientifiques et techniques. 
2 En effet, penser le format depuis ce point de vue suppose d'identifier un certain rapport à la réalité envisagée fondamentalement comme hétérogène. S'il fallait tirer un postulat philosophique de cette attitude, qui déterminerait aussi une esthétique, il faudrait dire que, selon cette approche, la réalité matérielle ou symbolique ne saurait se rapporter à une substance unique, ni à une forme ou une structure uniques (postulat qui mobiliserait beaucoup d'arguments, de la métaphysique à la physique théorique en passant par l'anthropologie). Au sein de cette réalité, le format est ce qui apparaît comme le moyen de circulation privilégié. Pourquoi ? Parce que cette circulation ne peut recourir à aucun véhicule universel, aucun langage qui pourrait prétendre à une sorte d'opérativité absolue, à l'inverse d'une certaine conception du médium, moyen matériel attaché à un art donné, que la modernité artistique a tendu à essentialiser dans sa quête d'un langage artistique auto-réflexif. Passer d'un médium à l'autre met au contraire l'accent sur ce qui se passe entre les médiums et sur la contingence et le caractère relatif de tel ou tel moyen matériel, dont l'opérativité dépend des paramètres d'un certain format. Le format est en effet ce qu'on rencontre, dans un monde fini, lorsqu'on porte l'attention précisément aux conditions locales, topiques et particulières de fonctionnement des langages, des médiums et des formes et plus généralement des systèmes symboliques, tout autant que des systèmes techniques et matériels. On peut ne pas voir le format d'un système quelconque par lequel une information nous est transmise ou par lequel une expérience nous est permise, ou ne pas considérer (à tort sans doute) cette dimension de fonctionnement de l'information ou de condition de l'expérience comme pertinente pour la connaissance, l'action ou la création. Mais il n'en demeure pas moins qu'un format, nécessairement, est toujours à l'œuvre.

3 Nous entendons ici la notion de format comme un cadre matriciel et opératoire constitué de normes et de règles qui agencent des représentations et des pratiques, de façon relative à la fois du point de vue de chaque format particulier et du point de vue du rapport d'un format donné à ce qui lui est extérieur. Dans un format, les normes et les règles peuvent changer selon certains paramètres (caractère relatif interne), et d'autre part un format implique des logiques d'implémentation contextuelle, des règles de compatibilité et d'interopérabilité entre formats, ainsi que la détermination de la réalité qui peut être « opérée » ou œuvrée à travers lui, et sous quelle forme (caractère relatif externe). Une telle définition déploie certains termes que nous avons proposés par ailleurs, à savoir l'idée d'une « construction ou d'une convention qui norme des modes d'inscription, de codage, de traduction, de compatibilité, d'implémentation, d'exposition, de filtrage et d'usage de formes et d'informations » (Zerbib 2015a, 16). Le terme de format fait signe vers le domaine des standards techniques de l'industrie ainsi que vers les modalités de structuration des données en informatique, après avoir été lié traditionnellement aux questions d'échelle, de durée et de dimension propres aux arts. Cette expansion sémantique paraît hautement significative, et nous avons suggéré qu'en dépit de sa connotation instrumentale, accessoire, matérielle et pauvre symboliquement, le format constitue un enjeu clé de toute création contemporaine. Au point d'offrir une prise théorique suffisamment consistante pour permettre de définir le propre du "contemporain" dans les arts, selon une inflexion historique des pratiques et des discours qui relativise la centralité de la question moderne de la forme, sans qu'il soit pour autant nécessaire de verser dans une certaine esthétique anomique de la postmodernité. Ce que souligne la question du format est en effet un déplacement des enjeux normatifs en matière de création, plus qu'une perte de critères esthétiques (Zerbib 2015b). L'anomie esthétique qui semble parfois caractériser l'art contemporain 
tiendrait à cet égard à un effet retard des termes du jugement esthétique sur les normes effectivement à l'œuvre dans les processus de production artistique.

\section{Pluralisation des mondes et crise de la représentation}

Un faisceau de phénomènes convergents a produit une révélation progressive de ce plan de la réalité matérielle et symbolique délaissé par l'attention théorique. Mise au jour qui est, toujours, en même temps une mise à jour, autrement dit un update de la pensée qui modifie, dans une certaine mesure, le paradigme - ou le format épistémologique - à partir duquel nos raisonnements et nos gestes opèrent sur les idées, les formes et les choses. Il est ainsi utile de signaler, même succinctement, certaines des conditions d'émergence de la question du format, afin de mieux en mesurer la portée. Dans ce faisceau de phénomènes, nous pourrions distinguer trois ensembles. Le premier est de nature esthétique, le second technologique et le dernier politique. Le premier consiste en une certaine crise du formalisme. Autrement dit une situation de remise en question des valeurs développées au sein des théories formalistes dans le domaine esthétique et poétique. Cette crise peut être associée à la destitution de la pureté formelle, à la dissolution de l'autonomie de l'art moderniste, au décloisonnement des régimes d'écriture et à l'irruption de matériaux textuels ordinaires dans la littérature par exemple, ou encore à la nouvelle place de la culture populaire et commerciale dans les arts d'une manière générale. Les traits de cette crise du formalisme coïncident en partie avec un certain diagnostic historique formulé sous l'angle du « postmodernisme » (Jameson).

5 Le second ensemble regarde l'évolution technologique et la prégnance croissante dans tous les domaines de la pratique des processus d'informatisation, autrement dit de discrétisation, de calcul, de programmation et de mise en réseau. Outre le tournant que représente l'informatisation dans notre «écologie médiale» (Mersch 228), les technologies de l'information et de la communication soulignent de façon spécifique le rôle des interfaces, des conditions d'interopérabilité des langages et du paramétrages des dispositifs de traduction du continuum sensible en information, soit autant de processus, de conception, de décisions ou d'usages, qui se formulent au niveau des formats de l'information. À cet égard toute informatique est aussi, sinon d'abord, une formatique, autrement dit une technique portant sur les conditions opératoires de l'information numérique, qui commence notamment par définir les normes de codage ou de «représentation » des données (à travers, par exemple, la règle de conversion d'un caractère en une suite binaire de 0 et de 1 en fonction d'un nombre limité de bits, 8,16 ou 32).

6 Enfin, il faut ajouter à ces deux ensembles les phénomènes de fragmentation institutionnelle, sociale et culturelle. Non que les institutions politiques, les cultures ou les sociétés aient été un jour constituées d'ensembles monolithiques et homogènes. Mais les différences, les hiérarchies et les formes de dominations ou de conflictualité qui s'y manifestent ont tendu à être rapportées à des structures à vocation universelle et centralisée : systèmes de représentation politique, centralisme étatique, lutte des classes, anthropo-ethnocentrisme occidental, structures patriarcales des rapports sociaux de sexe et de genre... On ne peut nier que les grandes ruptures politiques, sociales, idéologiques et culturelles depuis le tournant du XXIe siècle soient liées à des processus de recomposition des structures, des cadres, des hiérarchies et des systèmes 
selon une dynamique qui n'implique pas la substitution d'un système à un autre mais la pluralisation des modèles et des systèmes de règles, mettant en jeu une pragmatique des compatibilités. Or, quand les structures centralisatrices vacillent, les formats prolifèrent, et inversement: la multiplication des formats fragilise les logiques unificatrices, standardisatrices et autoritaires des structures centralisatrices.

7 Cette pluralisation n'empêche pas de reconnaître la prégnance de certains rapports de force ni le fait que certaines situations sociales, par exemple, peuvent être surdéterminées par ces rapports de domination qui les traversent et se définissent à une autre échelle. Mais si l'on considère le fait que pour être saisie dans sa complexité et sa plasticité, la réalité sociale ne doit pas être abordée à travers le prisme d'un invariant structurel unique, alors apparaît le jeu d'imbrication des «cadres de l'expérience ", selon le concept d'Erving Goffman, qui constituent autant de formats construisant la réalité sociale (Goffman). Pour ne prendre qu'un exemple dans le champ qui nous concerne le plus directement, le monde de l'art contemporain se présente précisément sous les traits du polycentrisme et du pluralisme esthétique. D'un point de vue historique, ce polycentrisme est bien décrit par Béatrice Joyeux-Prunel par exemple (Joyeux-Prunel). Quant à la sociologie des «mondes de l'art» de Howard Becker (Becker), ou l'analyse des agencements dans la théorie de l'acteur-réseau (Akrich, Callon et Latour), elles insistent aussi, pour ne prendre que ces deux exemples, sur des recompositions qui vont dans le sens de l'articulation dynamique d'une réalité plurielle et décentrée, impliquant une analyse des fonctionnements sociaux en termes de connexions et de relations qui dessinent autant de chaînes opératoires hétérogènes.

8 En dépit des apparences, ces thèmes ne sont pas éloignés du geste d'un artiste qui, comme Boris Achour, utilisait en 2001 le format des jaquettes des vidéos de films, qu'il détournait pour introduire des références à Michel Foucault, à la musique des Destiny's Child ou à la grève des employés d'un McDonald, le tout dans une œuvre intitulée Cosmos, qui empruntait au roman de Witold Gombrowicz son titre et l'accent porté sur l'enjeu de l'organisation du chaos (Gombrowicz). Il s'agit bien là de repenser le réel sur un plan horizontal - le philosophe Gilles Deleuze dirait "un plan d'immanence » (Deleuze et Guattari 41) - où les différences, les fonctions, les circulations, les productions de sens, doivent être repensées à partir de leur effectivité et opérativité immanente, ou de leurs agencements et de leur productivité propre, et non depuis un point de surplomb, ou de fondation, central et unificateur.

9 Le critique et commissaire d'exposition François Piron défendait à propos de l'œuvre d'Achour la "possibilité d'une analogie entre son travail et la structure du roman de Gombrowicz qui, sous l'apparence d'un roman policier traditionnel, voire stéréotypé, mène une recherche plus abstraite sur le sens que l'on donne à des choses disparates et a priori insignifiantes. Il soutient l'idée que l'on peut travailler à partir de n'importe quoi, car ce qui importe, c'est la structure, l'organisation, le système mis en place pour nommer et rendre signifiants des éléments disparates " (Achour, Francblin et Piron, n.p.). Si ce qui compte n'est pas l'identité substantielle d'une chose, ni les hiérarchies ontologiques où s'inscriraient ces identités, et si le travail de l'art peut se jouer sur ce plan horizontal «à partir de n'importe quoi », on comprend que l'enjeu porte sur les formats du chaos, pour reprendre le terme de cet enjeu mentionné précédemment à propos du livre de Gombrowicz, une fois celui-ci reconnu sur fond de monde esthétique décloisonné. 


\section{Format et structure}

10 "Structure ", " organisation ", "système ", énumère le critique d'art... Il faudrait à ce sujet préciser que l'attention à la «structure » suppose de distinguer un sens ordinaire et un sens fort du terme, ou encore un sens pragmatique et un sens formaliste. Dans le premier sens, il s'agit d'une forme organisatrice, qui ne constitue pas nécessairement la loi fondamentale de ce qu'elle organise. Dans le sens fort, qui est celui du structuralisme dans les sciences humaines, la structure est la loi interne de ce qui est étudié, la forme abstraite qui fonde l'intelligibilité des éléments à travers les relations que la structure établit entre eux. À propos de l'approche structurale des études littéraires, dite poétique en ce sens spécifique, Tzvetan Todorov écrivait: "par opposition à l'interprétation d'œuvres particulières, elle ne cherche pas à nommer le sens mais vise la connaissance des lois générales qui président à la naissance de chaque œuvre. Mais par opposition à ces sciences que sont la psychologie, la sociologie, etc., elle cherche ces lois à l'intérieur de la littérature même. La poétique est donc une approche de la littérature à la fois 'abstraite' et 'interne' » (Todorov 19). Le théoricien de la littérature voulait ainsi distinguer la poétique structurale à la fois de « l'interprétation » qui s'intéresse au sens interne d'une œuvre mais pas à sa loi, et de la "science » qui s'intéresse à sa loi abstraite mais qui demeure externe à l'œuvre. La structure est donc une loi abstraite immanente à l'œuvre, elle est la forme fondamentale qui préside aux différentes formes apparentes de l'œuvre. En ce sens l'analyse tend à affranchir l'œuvre de ses lois de fonctionnement externes et contextuelles.

11 À la différence d'une telle lecture structuraliste, une approche en termes de formats, que l'on pourrait appeler, au besoin, formatiste, s'intéresse aux cadres fonctionnels des formes, tout en mettant l'accent sur leurs dehors et leurs rapports, sur l'hétéronomie des passages, des connexions, des traductions, des transductions. Elle infléchit le sens de la structure pour lui conférer une dimension relative et située, rapportant sa pure géométrie à un diagramme hétérogène qui connecte des langages et des plans différents de la réalité. Interfaces, filtres et «re-médiations» (Quintyn 2007, 17-65) priment alors sur la loi interne. En reprenant la distinction de Tzvetan Todorov entre l'interprétation (le sens interne), la science (la loi externe) et donc l'analyse structurale (la loi interne), il faudrait considérer qu'à travers la notion de format l'attention porte sur les règles des opérations situées. Le format est donc moins loi causale interne que raison pragmatique. C'est en effet pour pouvoir opérer d'une certaine manière et dans un certain contexte technique et pratique qu'un format est défini, depuis le format d'une page de livre imprimée en fonction de la taille des outils de presse, eux-mêmes conditionnés par certains usages, jusqu'au protocole de compression de l'image numérique dans le format "jpeg », en passant par la taille normalisée des containers permettant d'interopérer des bateaux, des camions et des trains dans le transport des marchandises sur les routes de la globalisation (Burch et Sekula). Le format prescrit comment une forme peut opérer, à partir de quelles conditions de mise en relation et d'activation, ou encore d'implémentation, pour reprendre un concept de Nelson Goodman à propos du fonctionnement d'une œuvre d'art (Goodman 63-68). De ce point de vue, le format ne dit pas la vérité de l'œuvre, son essence, ce en quoi elle est ce qu'elle est, ni ce que Todorov appelle sa loi interne; le format programme l'opération ou, mieux, l'opérabilité de l'œuvre. Opérabilité sans laquelle, à proprement parler, elle 
n'œuvre pas. L'opérabilité de l'œuvre constitue comme une règle de l'art, qui est d'autant plus implicite qu'elle semble ne porter que sur des évidences techniques, secondaires au regard de la prévalence des normes esthétiques et des débats théoriques ou critiques que ces normes suscitent.

La dimension normative d'un format peut encore être précisée au regard de la différence entre une règle et une loi causale. Cette différence éclaire deux manières de se rapporter à l'idée de structure. Sur ce point, Jean-Pierre Cometti explique qu'une règle implique " une manière correcte et une manière incorrecte de faire ce que l'on fait », ce qui n'a pas de sens dans le domaine des causes :

Apparemment, une telle distinction est de nature à s'appliquer à tout ce qui mérite à nos yeux le nom de règle, $\mathrm{y}$ compris précisément à ces règles que postulent les approches de type structural en sociologie ou en anthropologie, comme en linguistique. Mais on peut aussi avoir de bonnes raisons de penser que la capacité d'y accéder par la réflexion en est aussi un critère, ce qui constitue l'un des points de discorde, dans les sciences sociales, entre les conceptions qui privilégient les structures et leur subordonnent les comportements, les actes et les croyances, et celles qui se montrent plus attentives aux processus d'interaction qui semblent en être constitutifs. (Cometti 15)

13 Ces deux «façons d'accéder » à une structure recouvrent en gros la différence entre une approche formaliste et une approche de type pragmatiste. Dans le premier cas, nous avons affaire à la forme substantielle de l'objet d'étude, ou à sa loi structurale interne, qui dit ce qu'il est ; dans le second cas, au format situé de l'objet, autrement dit à une règle opératoire contextuelle, qui paramètre ce qu'il fait.

14 Afin d'éclairer cette distinction à partir du champ de l'art, prenons l'exemple de l'œuvre picturale. Nous pouvons considérer qu'une image peinte doit répondre à certaines règles de mise en espace et de monstration, ainsi qu'à des dispositifs matériels d'inscription qui conditionnent sa visibilité, de même qu'à des conditions esthétiques de cadrage qui délimitent le champ d'exercice de cette visibilité. Ces différentes règles définissent, au fond, le format "tableau » de la forme picturale. Au sein de ce format la peinture opère correctement lorsque, par exemple, le tableau est fixé sur un plan perpendiculaire au plan horizontal où s'érige le point de vue du spectateur, la face peinte tournée vers lui. Le même tableau retourné, décroché, se retrouvant à boucher une vitre cassée, ou une toile de Rembrandt servant de couverture, pour reprendre un célèbre exemple de Nelson Goodman (Goodman 68), peut à la limite continuer à être considéré comme une œuvre d'art, mais ne fonctionnerait plus comme telle, au regard du format standard de la peinture.

\section{Un processus historique d'explicitation}

Comme tout ensemble de règles, un tel format peut fonctionner de façon explicite ou implicite. Par ailleurs, comme le montre Robert Brandom, « en tant que règles, les normes explicites présupposent des normes implicites dans des pratiques ", celles-ci déterminant comment les normes sont correctement appliquées (Brandom 20). Des conventions, des manières de faire, des habitudes, donc autant de normes implicites, régulent ainsi l'application des normes explicites.

16 Ainsi ne pas voir le dispositif qui nous donne à voir, ou rendre transparent le format qui nous fait accéder à la forme et permet par exemple l'accomplissement de la norme esthétique explicite du Beau, facilite le transport dans l'espace propre de l'œuvre et la 
projection du regard, dans la perspective ouverte par la fenêtre du tableau. Laisser le format de l'œuvre au niveau d'une condition instrumentale et technique, ou d'une catégorie générique qui détermine simplement une classification des types d'œuvres (comme dans le cas des formats de tableau qui correspondaient classiquement à l'importance de certains sujets), participe ainsi d'une règle du jeu relativement implicite de l'expérience de la peinture classique. Il en va de même de la règle autoinstituée du génie, qui masque le jeu des normes implicites à partir duquel l'invention de nouvelles règles peut avoir lieu. "À cet égard », remarque Jean-Pierre Cometti, «la manière dont l'art a conquis son statut autonome, dans nos sociétés, est la source de bien des confusions. Kant, bien qu'il tienne le concept d'art pour solidaire du concept de règle - 'tout art possède ses règles' - en est néanmoins victime lorsqu'il confie au génie le soin de donner sa règle à l'art » (Cometti 120).

Or, en deçà de l'aventure historique des formes picturales, des théories qui l'ont accompagnée et des normes esthétiques qui en ont nourri le jugement, ne peut-on pas observer comme une sorte d'explicitation progressive de cette règle du jeu de l'art implicite en quoi consiste le format de l'œuvre, sur un plan non seulement ontologique mais aussi esthétique? Une fois le tableau "instauré », pour reprendre les termes de Victor Stoichita (Stoichita), c'est-à-dire non seulement déterminé sous certaines conditions matérielles mais également conceptuelles, à travers en particulier la réflexivité du tableau dans la peinture, comme on peut le voir de façon célèbre dans Les Ménines de Velásquez (dont Michel Foucault faisait le modèle de «la représentation de la représentation classique ", Foucault 31), la modernité a fait de ses conditions visuelles d'instauration le motif d'une réflexion picturale. Cette réflexion a été néanmoins longtemps dominée par une essentialisation formaliste, au sens où la réflexion sur les conditions du langage pictural a tendu à interroger une structure fondamentale, sinon une loi transcendantale, comme on le voit dans les analyses du théoricien de l'art américain Clement Greenberg (Greenberg). Autrement dit, la réflexivité du tableau est passée d'une métaphysique de la représentation (essence ou idée de la représentation, comme réglage du rapport entre idée et forme, qui se donne à voir dans la représentation même) à un formalisme abstrait (essence du langage pictural qui se donne à voir en lui-même et pour lui-même).

Or il est une autre orientation de la réflexivité du tableau qui non seulement fait émerger le rôle du format $d u$ tableau, mais encore la fonction et les usages du format "tableau ». Cette autre orientation suppose de considérer le tableau comme un cadre opératoire, de le faire fonctionner et de le donner à percevoir comme tel. Des degrés divers peuvent être identifiés dans ce régime formatiste du tableau, dans lesquels nous pourrions distinguer, par exemple, Kandinsky qui affirme la dimension matricielle du format sans pour autant en faire l'enjeu de l'expérience esthétique en tant que tel dans sa théorie du "Plan originel » (Kandinsky), de Claude Rutault qui prolonge à son terme l'aventure formaliste jusqu'au « suicide » de la peinture en exposant des toiles réduites à des séries de formats monochromes vides, parfois simplement stockées dans des rangements ad hoc ou empilées en fonction de leur dimension ${ }^{1}$, et qui produisent l'expérience mélancolique d'une ultime esthétique formaliste, celle d'un formalisme du format, qui rencontre alors l'ambigüité, sinon la contradiction, de ce qui précisément ne peut se réduire à une forme autoréférente et qui, en un sens, rejoint les variations inscrites dans le projet de l'artiste intitulé Des histoires sans fin (Breuil). 
19 À la différence de ces deux figures très liées au modernisme pictural et situées à deux extrémités de son développement historique, il faut se tourner vers des artistes contemporains comme Matthew Barney pour voir le format fonctionner comme l'enjeu non seulement matriciel mais aussi esthétique de l'œuvre. Les travaux de la série Drawing restraint (commencée en 1987) illustrent parfaitement ce basculement. Dans les premières œuvres de cette série, on voit l'artiste soumis à des contraintes physiques et techniques qui conditionnent l'accomplissement d'un geste graphique ${ }^{2}$. Dans un espace blanc, qui correspondrait en principe à une salle d'exposition destinée à montrer des dessins encadrés et accrochés aux murs, nous voyons des planches, des cordes, des sangles et autres accessoires évoquant une salle de gymnastique qui aurait été mise sens-dessus-dessous. L'artiste, accroché lui-même au mur au milieu de ces éléments, doit accomplir d'importants efforts pour pouvoir inscrire sur une feuille de papier, dont il ne peut pas se rapprocher, des lignes tracées à distance et à bout de bras à l'aide d'un long manche pourvu à son extrémité d'un outil graphique.

Cette œuvre montre très bien que l'enjeu esthétique ne tient pas à la forme graphique elle-même. Celle-ci résulte simplement, dans ce jeu de dessin d'obstacles (comme on parle de course d'obstacles), des contraintes opératoires que l'artiste s'est imposées, ces contraintes possédant d'ailleurs autant d'importance dans l'intitulé de l'œuvre que le dessin lui-même (l'œuvre est autant drawing que restraint). La forme graphique est donc loin de constituer la finalité de l'œuvre. Elle n'affirme en aucune façon sa présence comme étant l'indice d'un événement autoréférent qui absorberait et nierait tout son processus, au contraire de ce que fait advenir un "zip» de Barnett Newman, pour prendre un exemple diamétralement opposé à cette logique, emblème éclairant du formalisme abstrait.

21 Dans Drawing restraint, la trace d'un processus fait du cadre de l'œuvre et de son activation son véritable enjeu. Un peu comme si le cadre du dessin était devenu la scène de sa performance, dont le corps de l'artiste serait le médium. Matthew Barney a défini des règles du jeu, une série de contraintes, de dispositifs techniques et matériels qui restreignent les possibilités du geste graphique, l'empêchant de coïncider avec l'exercice d'une libre expressivité autant qu'avec le respect d'un impératif de pureté formelle. C'est bien dans le cadre matriciel de l'œuvre, dans le réglage de ce qui peut la produire, et dans ses conditions opératoires, que réside l'enjeu esthétique. C'est ce format de la forme graphique qui est donné à voir, apprécier et réfléchir. Il est le fruit de décisions et de dispositions qui ne sont plus transparentes, étant explicitées par l'effort physique et l'excès instrumental de sa mise en œuvre.

Une œuvre comme celle de Frank Leibovici illustre un autre type de processus d'explicitation esthétique des enjeux du format dans l'art contemporain. Elle montre en particulier qu'une pragmatique du format peut expérimenter un lien nouveau à la réalité sociale et politique. L'artiste prend pour matériau de ses opérations plastiques ce qu'il appelle des "documents poétiques». Ces matériaux sont comme des représentations d'une réalité, cristallisées dans des formes d'énonciation, des discours, des images, des pratiques, produites par certains collectifs ou individus à propos de certaines questions ou problèmes, et que l'artiste décide de "performer », c'est-à-dire d'activer d'une manière qui en épuise les contenus, afin de donner à percevoir les cadres constitutifs de ces représentations et énonciations. Cette performation, dirionsnous, équivaut à une opération de traversée des formats, l'artiste faisant passer par exemple un diaporama PowerPoint produit par le gouvernement des États-Unis dans 
un livret d'opéra appelé à être chanté par un groupe de non-musiciens. Les contenus représentationnels d'un document donné sont ici redistribués esthétiquement et politiquement, transformatés de manière à modifier notre rapport à la réalité sociale en question (Zerbib 2018).

Le processus d'explicitation du format dans les arts se manifeste selon des logiques plus ou moins performatives et plus ou moins formalistes, ou plutôt paraformalistes, pour ne pas confondre cet aspect avec la revendication moderniste. De même que nous distinguions le formalisme du format chez Claude Rutault au regard des contraintes "performées" par Matthew Barney, nous pourrions distinguer, face au document «performé » par Franck Leibovici, les expositions du groupe Experimental Jetset qui mettent en espace des formats rendus visibles pour eux-mêmes, comme dans Lost formats preservation society $(2000)^{3}$. Toute mise en espace, tout dispositif de visibilité relève cependant en un sens d'une performance scénologique, ou d'un " acte d'image " qui équivaut sur le plan de l'expérience visuelle à la performativité d'un acte de langage (Bredekamp). Une forme géométrique peut prétendre se soustraire, relativement, aux conditions matérielles, contextuelles, techniques, sociales et symboliques de son dessin, car elle trouve sa plus grande effectivité opératoire dans sa nature d'idéalité abstraite. En revanche, un format $\mathrm{A} 4$ ne peut pas prétendre à cette abstraction. Son opérativité s'exprime plus dans des logiques de transaction, de décontextualisation et de recontextualisation. Dans les différentes modalités d'explicitation du format dans les arts, les distinctions relèvent de variations des critères pragmatiques et opératoires, car la question du format, même lorsqu'elle est exhibée pour elle-même, nous oblige à considérer ce par quoi l'œuvre opère.

\section{Toute œuvre est un opérat}

24 De nombreuses théories contemporaines portant sur les arts de la modernité tardive, ou post-modernistes sinon post-modernes, insistent sur des basculements touchant notamment le passage de l'objet au contexte, de l'œuvre exposée à la relation, de l'art autonome à la réalité ordinaire, de la forme matérielle au concept ou au geste, de l'expérience esthétique au jeu institutionnel ou à l'analyse linguistique, du sublime à la marchandisation, de la contemplation à la médiation, etc. Outre l'intérêt analytique que peuvent évidemment présenter ces théories dans notre approche de telle ou telle pratique artistique, ou pour notre connaissance du fonctionnement du monde de l'art, ces tentatives de rendre compte du contemporain dans les arts présentent parfois l'inconvénient de nous laisser penser que le contemporain se définit comme un changement d'époque qui possède comme contenu positif de nouvelles préoccupations artistiques, de nouveaux styles, de nouveaux thèmes et de nouveaux contextes qui rompent avec l'époque antérieure, nous incitant à proclamer obsolètes les anciennes théories.

Or, plutôt qu'affronter seulement la problématique qui consiste à comprendre comment essentialiser des différences observables dans les pratiques afin de marquer un changement paradigmatique dans l'histoire des arts, il nous semble important de réinterroger la logique qui paraît traverser l'histoire, ses périodes et ses styles, et dont l'explicitation du format serait pour la période contemporaine la manifestation. Ainsi il ne suffit pas de distinguer l'ère moderne de la forme de l'ère contemporaine du format, mais de comprendre de quelle question transhistorique le format est le nom. Or, nous 
pouvons faire l'hypothèse qu'il s'agit d'une question portant fondamentalement sur les puissances de l'œuvre. Autrement dit, sur ce qui fait qu'une œuvre œuvre, sur l'œuvrer de l'œuvre, ce qui implique qu'un artefact possède en soi la puissance d'œuvrer tout en étant le produit d'un travail humain. Il n'est pas possible de développer ici cette hypothèse mais on peut observer que la question du format prolonge un procès en immanence de cette puissance, c'est-à-dire une attitude consistant à chercher dans l'œuvre même ce qui peut lui conférer le moyen d'une d'autonomie, d'une capacité d'agir, ou d'une agentivité propre, dirions-nous dans les termes de l'anthropologie de l'art, que cette capacité d'agir soit, par exemple, magique, liturgique, mystique, métaphysique, psychologique, politique ou critique. Si nous ressaisissons à cette aune le contraste souligné précédemment entre modernité et contemporanéité, il apparaît que ce n'est plus dans la pureté d'une forme que l'œuvre cherche son autonomie, mais dans le paradoxe d'une "hyper-hétéronormativité ». Cette hyper-hétéronormativité qualifie une traversée de l'hétérogénéité des normes et des règles susceptible de produire un type paradoxal d'autonomie, dans lequel la capacité d'agir ne naît pas du fait de se gouverner selon ses propres lois (selon une définition nominale de l'autonomie) mais du fait de se déplacer entre les normes, en les performant de telle sorte qu'elles apparaissent plastiques, relatives et contingentes ${ }^{4}$.

Retrouvant ici les enjeux d'hétérogénéité, de transitivité et de normativité que nous avions mentionnés au début de ce texte, nous pouvons à présent mieux comprendre leur articulation dynamique. Un format agence des possibilités opératoires. En tant que tel, il est donc traversé par des forces qui l'activent, en même temps qu'il conditionne la performance de ces forces et les formes dans lesquelles elles peuvent se manifester. Un format agence ainsi d'une manière spécifique des matériaux, des forces et des formes symboliques qui peuvent, qui ont pu, et qui pourront, être agencés autrement ${ }^{5}$. Dans le domaine symbolique comme en physique, la création relève assurément plus de la recomposition d'une quantité donnée de matière et d'énergie que d'une apparition géniale ex nihilo. De sorte que pour comprendre les processus de création ou, mieux, de formativité, selon le terme du philosophe Luigi Pareyson (Pareyson), il apparait incontournable d'observer le jeu engagé entre certains cadres matriciels et leur activation, entre structure et performance, ou entre un format et l'opération qu'il conditionne et qui, en retour, peut le transformer, selon l'agencement des dynamiques qui le traversent.

La réflexion sur le format gagne ici à inscrire l'analyse pragmatique dans une théorie générale des opérations, que nous pouvons appeler, d'après le concept de Gilbert Simondon, une allagmatique. L'allagmatique est en effet pour le philosophe de la technique "la théorie des opérations ». L'opération étant «ce qui fait apparaître une structure ou qui modifie une structure " (Simondon 559) ${ }^{6}$. L'allagmatique désigne à ce titre une science du changement qui analyse la relation entre opération et structure. La notion de format désigne précisément, avons-nous montré plus haut, une structure de nature plastique. En matière de philosophie de l'art, le format relève donc d'une telle théorie des opérations, sorte d'allagmatique esthétique et symbolique où la singularité d'une forme doit être pensée dans les conditions de la technique. Toute œuvre, a fortiori celles relevant d'une esthétique « opérationnaliste » (Quintyn 2017, 41) mais également au-delà, depuis le dessin griffonné sur un coin de nappe jusqu'à l'opéra wagnérien, apparaît à cet égard comme ce que nous proposons de nommer un opérat, autrement dit le produit d'une opération paramétrée par un format technico-artistique et susceptible d'opérer en tant que tel sur un plan symbolique et esthétique. Cuvre et opération, qui 
partagent une même étymologie $\mathrm{e}^{7}$, retrouvent à nos yeux et à travers le rôle du format dans les pratiques contemporaines un plan d'analyse commun, nous incitant à reconsidérer la notion d'œuvre dans ses dimensions et puissances opératoires.

\section{BIBLIOGRAPHIE}

\section{Ouvrages cités}

ACHOUR, Boris, FRANCBLIN Catherine et François PIRON. « L'Art d'organiser le chaos avec Boris Achour et François Piron ». Entretiens sur l'art. Fondation d'entreprise Ricard (5 avril 2006). URL : https://www.paris-art.com/entretiens-sur-lart-boris-achour/ (page consultée le 7 juin 2021).

AKRICH, Madeleine, CALLON, Michel et Bruno LATOUR. Sociologie de la traduction. Textes fondateurs. Paris : Les Presses de Mines ParisTech (coll. « sciences sociales »), 2006.

BECKER, Howard S. Les Mondes de l'art. Trad. J. Bouniort. Paris : Flammarion, 1988.

BRANDOM, Robert. Making it explicit: Reasoning, Representing, and Discursive Commitment. Cambridge MA. : Harvard University Press, 1994.

BREDEKAMP, Horst. Théorie de l'acte d'image. Trad. Frédéric Joly et Yves Sintomer. Paris : La Découverte, 2015.

BREUIL, Marie-Hélène. Claude Rutault. L'inventaire. Genève : Mamco, 2015.

BURCH, Noël et Allan SEKULA. The Forgotten Space. Film coul., 110 min. Doc.Eye Film, WILDart Film, 2009

COMETTI, Jean-Pierre. Qu'est-ce qu'une règle ? Paris : Vrin, 2011.

DELEUZE, Gilles et Felix GUATTARI. Qu'est-ce que la philosophie ? Paris : Minuit, 1991.

FOUCAULT, Michel. Les mots et les choses. Paris : NRF Gallimard (coll. Bibliothèque des sciences humaines), 1966.

GOFFMAN, Erving. Les Cadres de l'expérience. Trad. Isaac Joseph, Michel Dartevelle et Pascale Joseph. Paris : Éditions de Minuit, 1991.

GOMBROWICZ, Witold. Cosmos. Trad. Georges Sédir. Paris : Denoël, 1966.

GOODMAN, Nelson. L'Art en théorie et en action. Trad. Jean-Pierre Cometti et Roger Pouivet. Paris : Gallimard (coll. Folio), 2009.

GREENBERG, Clement. «Vers un nouveau Laocoon ». Art en théorie 1900-1990. Une anthologie. Ed. Charles Harrison et Paul Wood. Paris : Hazan, 1997. 614-620.

JAMESON, Fredric. Le Postmodernisme ou la logique culturelle du capitalisme tardif. Trad. Florence Nevoltry. Paris : École nationale supérieure des beaux-arts de Paris, 2011.

JOYEUX-PRUNEL, Béatrice. Naissance de l'art contemporain. 1945-1970. Une histoire mondiale. Paris : Éditions du CNRS, 2021. 
KANDINSKY, Wassily. Point et ligne sur plan. Contribution à l'analyse des éléments de la peinture. Trad. Suzanne et Jean Leppien. Paris : Gallimard (coll. Folio), 1991. 143-151.

MERSCH, Dieter. Théorie des médias. Une introduction. Trad. Stéphanie Baumann, Philippe Farah et Emmanuel Alloa. Dijon : Presses du réel (coll. Médias/Théories), 2018.

PAREYSON, Luigi. Esthétique. Théorie de la formativité. Trad. Gilles Tiberghien. Paris : Rue d'Ulm (coll. Aesthetica), 2007.

QUILLET, Pierre. Ernst Cassirer. Paris : Ellipses, 2001.

QUINTYN, Olivier. Dispositifs/Dislocations. Paris : Questions théoriques (coll. « Forbidden Beach »), 2007.

QUINTYN, Olivier. Implémentations/Implantations. Paris : Questions théoriques (coll. Ruby Theory), 2017.

SIMONDON, Gilbert. L'Individuation à la lumière des notions de forme et d'information. Grenoble : Jérôme Millon, 2005.

STOICHITA, Victor. L'Instauration du tableau. Métapeinture à l'aube des temps modernes. Seconde éd. revue et corrigée, Paris : Librairie Droz, 1999.

TODOROV, Tsvetan. Poétique. Qu'est-ce que le structuralisme ? (2). Paris : Seuil (coll. « Points »), 1973. ZERBIB, David. « Voulez-vous enregistrer les modifications ? ». In Octavo. Des formats de l'art. Ed. David Zerbib. Dijon : Les Presses du Réel / ESAAA, 2015a. 13-19.

ZERBIB, David. « L’Ère du format ». In Octavo. Des formats de l'art. Ed. David Zerbib. Dijon : Les Presses du Réel / ESAAA, 2015b. 339-364.

ZERBIB, David. «Comment trans-formater la réalité ? Franck Leibovici et les usages du document ». Une Poétique pragmatiste. Considérations sur l'œuvre de Franck Leibovici. Ed. Rahma Kazam. Dijon : Presses du réel, 2018. 46-58.

\section{NOTES}

1. «Exposition-Suicide », Galerie Daniel Perrotin, Paris (8 janvier - 26 février 2011) : https://www.perrotin.com/fr/exhibitions/claude_rutault-exposition-suicide/763 (page consultée le 16 juin 2021).

2. Voir Drawing restraint 2 (1988) : https://www.frieze.com/article/matthew-barney-1 (page consultée le 16 juin 2021).

3. Voir https://www.flickr.com/photos/fotomuseum_ch/21802451629 (page consultée le 16 juin 2021).

4. Un exemple très simple d'un tel processus se trouve dans certaines performances qui traversent les normes de genre et d'identité sexuée. De ce point de vue, il y a une différence entre, d'un côté, revendiquer des droits et une autonomie attachée à une identité donnée, et, de l'autre, jouer le jeu de différentes identités, traverser les genres, donner à voir l'accomplissement de la norme d'une manière qui rend inopérant tout fondement essentialiste de telle ou telle norme.

5. Forces et formes symboliques qui se définissent très simplement, en suivant sur ce point la Philosophie des formes symboliques de Ernst Cassirer, comme une dynamique d'expressivité qui trouve sa réalisation dans des systèmes de signes (Quillet 13). 
6. «Elle est, dans l'ordre des sciences, symétrique à la théorie des structures, constituée par un ensemble systématisé de sciences particulières : astronomie, physique, chimie, biologie » (Simondon 559). Le terme allagmatique est formé à partir du grec allassein qui signifie « changer».

7. Comme le rappelle le Trésor de la Langue Française: «Du lat. opera, à l'orig. plur. de opus, operis, 'ouvrage, acte, travail', utilisé dep. Plaute comme fém. sing. au sens de 'travail, activité', et qui subsiste dans presque toutes les lang. rom.»

\section{RÉSUMÉS}

Dans cet article nous défendons l'idée que la question du « format » constitue un enjeu esthétique central des pratiques artistiques contemporaines, susceptible de caractériser le propre du « contemporain » dans les arts, par contraste avec la question de la «forme » dans la modernité. Défini comme cadre matriciel et opératoire composé de normes et de règles qui agencent des représentations et des pratiques, conditionne le fonctionnement de formes et de forces symboliques en permettant leur expérience et leur usage, le format infléchit l'analyse des formes et des structures en un sens pragmatiste et situé. Loin de soumettre ainsi les œuvres à des lois externes, le jeu hétérogène des formats nous incite à observer un nouveau type d'autonomie, à partir ce que nous appelons une « hyper-héteronormativité ». D'autre part, la question du format nous suggère ici de repenser l'œuvre à travers son "opération ", de manière à comprendre, notamment, sa capacité d'agir dans les conditions données de la technique.

In this article we argue that the issue of "format" is a key aesthetic principle in contemporary artistic practices, central enough to characterize the "contemporary" in the arts, in contrast to the question of "form" in Modernity. Defined as a matrix and operatory framework, made up of norms and rules that arrange representations and practices, governing the functioning of symbolic forms and forces, allowing their experience and their uses, the format modifies the analysis of forms and structures in a pragmatist and situated way. Far from submitting artworks to external laws, the heterogenic play of formats leads us to observe a new kind of autonomy, based on what we propose to call a "hyper-heteronormativity". Furthermore, the issue of format allows us to rethink the artwork through its dynamic of "operation", in order to understand, among other things, its capacity to act in given technical conditions.

\section{INDEX}

Mots-clés : format, esthétique, art contemporain, règle, structure, œuvre, opération, formalisme, pragmatisme, allagmatique

Keywords : format, aesthetics, contemporary art, rules, structure, artwork, operation, formalism, pragmatism, allagmatic 


\section{AUTEUR}

\section{DAVID ZERBIB}

HEAD-Haute École d'Art et de Design de Genève

David Zerbib est maître d'enseignement en Philosophie de l'art à la HEAD-Haute École d'Art et de Design de Genève, et enseigne également à l'ESAAA-Ecole Supérieure d'Art Annecy Alpes.

Membre associé du Centre d'Histoire des Philosophies modernes de l'Université de Paris 1

Panthéon-Sorbonne, ses recherches portent sur l'esthétique contemporaine, et en particulier sur les questions de performance et de performativité, ainsi que sur la notion de format. Il a notamment publié Performance Studies in Motion. International perspectives and practices in the twenty first century (avec Ati Citron et Sharon Aronson-Lehavi, Bloomsbury, Londres, 2014) et In octavo. Des formats de l'art, (Presses du réel - ESAAA, 2015). Il dirige également l'édition de Helmuth Plessner, Anthropologie philosophique de l'acteur. Imitation, expression et existence humaine (Éditions Paris Nanterre, coll. « Idées ", à paraître). 